\title{
Erratum to: An Integrative Analysis of microRNA and mRNA Profiling in CML Stem Cells
}

\author{
Farah J. Nassar, Rabab El Eit, and Rihab Nasr
}

Shaoguang Li and Haojian Zhang (eds.), Chronic Myeloid Leukemia: Methods and Protocols, Methods in Molecular Biology, vol. 1465, DOI 10.1007/978-1-4939-4011-0_18,

(c) Springer Science+Business Media New York 2016

DOI 10.1007/978-1-4939-4011-0_18

The old figure was inadvertently placed in the final files and so the below updated figure must replace the existing figure in chapter 18.

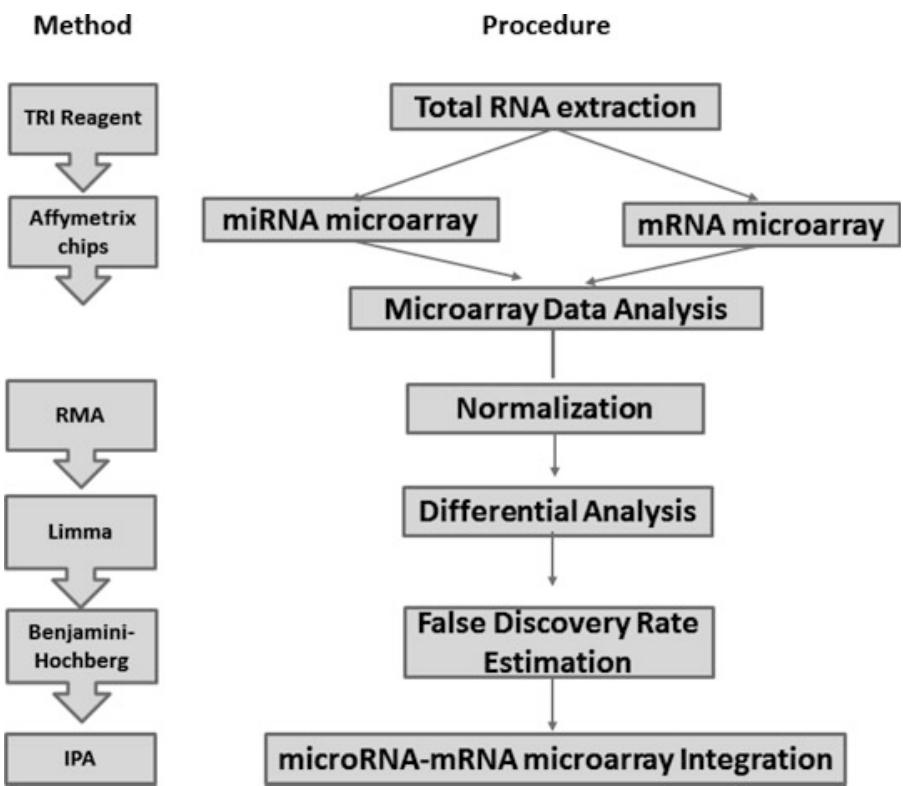

The online version of the original chapter can be found under http://dx.doi.org/10.1007/978-1-4939-4011-0_18

Shaoguang Li and Haojian Zhang (eds.), Chronic Myeloid Leukemia: Methods and Protocols, Methods in Molecular Biology, vol. 1465, DOI 10.1007/978-1-4939-4011-0_20,

(C) Springer Science+Business Media New York 2016 